\title{
Early Evolution of Vertical Vorticity in a Numerically Simulated Idealized Convective Line
}

\author{
Thomas A. Cram and Michael T. Montgomery \\ Department of Atmospheric Science, Colorado State University, Fort Collins, Colorado \\ Rolf F. A. HeRTENSTEIN* \\ Cooperative Institute for Research in the Atmosphere, Colorado State University, Fort Collins, Colorado
}

(Manuscript received 21 August 2000, in final form 3 December 2001)

ABSTRACT

\begin{abstract}
The generation and organization of mesoscale convective vortices (MCVs) is a recurring theme in midlatitude and tropical meteorology during the warm season. In this work a simulation of a finite-length idealized convective line in a westerly shear environment is investigated in the absence of ambient vertical vorticity. An asymmetry in average vertical vorticity forms rapidly at early times in the present simulation. This study focuses on the formation and organization of vertical vorticity at these early simulation times. Previous simulations suggest that tilting of either ambient or storm-generated horizontal vorticity is the primary mechanism responsible for the formation, organization, and maintenance of MCVs. This study confirms recent work regarding the generation of vertical vorticity at early times in the simulation. A Lagrangian budget analysis of the vertical vorticity equation, however, shows that vorticity convergence becomes a comparable, and at times dominant, mechanism for the enhancement and long-term organization of vertical vorticity early in the simulation. Despite differences in the initial ambient horizontal vorticity, hodograph, and convective available potential energy, the Lagrangian budget analysis in the present midlatitude case is consistent with the Lagrangian budget results of a previous tropical squall line simulation. The study of idealized convective lines in midlatitude environmental conditions therefore provide valuable insight into understanding vertical vorticity production in tropical squall lines and their potential relevance to tropical cyclogenesis.
\end{abstract}

\section{Introduction}

The role of mesoscale convective vortices (MCVs) in atmospheric dynamics is quite diverse. Frequently a by-product of mesoscale convective systems (MCSs), their mesoscale vertical vorticity structure and their association with the generation of new convective cells and strong surface winds produce environments conducive to cyclogenesis in both midlatitude and tropical regions during the warm season (Bosart and Sanders 1981; Zhang and Fritsch 1987). The presence of mesoscale vortical circulations in a finite-length convective line were first noted by Fujita (1978) in his pioneering study of downbursts and intense midlatitude bow and hook echoes. Often associated with damaging surface winds, such phenomena are characterized by "bookend

*Current affiliation: Colorado Research Associates, Boulder, Colorado.

Corresponding author address: Thomas A. Cram, Department of Atmospheric Science, Colorado State University, Fort Collins, CO 80523.

E-mail: tcram@eliassen.atmos.colostate.edu vortices" at the ends of the leading convective line (Weisman 1993), and these vortices can trigger new convective cells and produce background mesoscale vorticity, a favorable ingredient for tornado genesis. Recent work has suggested that line-end vortices of a finitelength convective line may be produced by the tilting of either ambient or storm-generated horizontal vorticity (Weisman 1993; Davis and Weisman 1994, hereafter DW94; Skamarock et al. 1994, hereafter SWK; Weisman and Davis 1998).

The mesoscale vortices simulated by DW94 in their weak-shear simulations were argued to form via the upward tilting of storm-generated horizontal vorticity, which was produced in the interface between the leading-line updrafts and the cold pool, by the front-to-rear ascending current. By assuming horizontal temperature perturbations are small in comparison to heating rates in the convective updrafts, DW94 established a relationship between the potential vorticity (PV) and vertical vorticity fluxes in which a convergence of the PV flux translates to a convergence of the vertical vorticity flux. Only $2 \mathrm{~h}$ into their simulation, a dipolar signature was seen in the PV flux, with convergence forming at the north end and divergence at the south end of the 
convective line. The front-to-rear inflow at this time in their simulation, however, was not well established, thus it was argued that the vorticity asymmetry at this time resulted from downward tilting of the ambient westerly shear by cooling downdrafts at low levels. By $3 \mathrm{~h}$, negative horizontal vorticity had developed between the front-to-rear ascending current and the rear inflow jet, at which time the mesoscale vortices assumed a more distinct, organized circulation. This suggests the importance of the front-to-rear and rear inflow currents, as it allows for the upward tilting of the storm-generated easterly shear to occur.

Using an identical ambient shear profile and convective available potential energy (CAPE) as the weakshear simulation in DW94, SWK identified three different sources of vertical vorticity production in a similar simulation to that of DW94. The first source, tilting of ambient horizontal vorticity by the initial convective updrafts, leads to a noisy signal in the vertical vorticity field due to the effects of the initial convection and gravity waves. The other two sources produce vertical vorticity via tilting of ambient westerly shear and tilting of westerly horizontal shear associated with the cold outflow, both by the descending rear inflow current. These two tilting mechanisms produced a coherent structure in the vertical vorticity field. At $6 \mathrm{~h}$ in their non-Coriolis simulation, the Eulerian plot of the tilting tendency term at $z=3 \mathrm{~km}$ was noisy, showing no clear signal of positive and negative vertical vorticity production north and south of the convective line center, respectively. The Eulerian view of the vorticity convergence tendency term at the same time was more organized and consistent with the asymmetric structure in vertical vorticity, suggesting the importance of vorticity convergence at later times.

Utilizing the flux form of the vertical vorticity equation, Weisman and Davis (1998) showed that the net vertical flux of horizontal vorticity along the centerline of the system provides the source for system-scale vortices at the line ends. This analysis of the vertical vorticity equation illustrates how cyclonic and anticyclonic vortices at the north and south ends of the convective line, respectively, can form from either a localized updraft in easterly shear or a localized downdraft in westerly shear. In conditions of stronger and deeper shear, vortices were also seen to result from ambient horizontal vorticity being tilted in the updrafts and downdrafts of supercellular convective systems.

The strength of low-level shear and the cold pool have been demonstrated to be important in determining what type of tilting occurs. Rotunno et al. (1988) described how sufficient low-level shear will counter the oppositely directed shear associated with the cold pool outflow, thus allowing new convective cell growth to occur at the cold pool boundary. The generation of vigorous convection and subsequent long life of the convective line is unlikely in environments with weak or no ambient shear since an optimal "balance" between the cold pool circulation and ambient shear is not achieved. For strong low-level shear, the bow echo simulations of Weisman (1993) showed that the main generator of vertical vorticity is tilting of ambient shear by updraft-downdraft couplets associated with more intense convective cells. The strong, deep-shear simulation of Weisman and Davis (1998) verifies this conclusion. The tilting of baroclinically generated negative horizontal vorticity seen in DW94, SWK, and Weisman and Davis (1998) is not as important for these strong, deep shear cases, simply because stronger shear inhibits the development of negative horizontal vorticity in the system and also because more positive horizontal vorticity exists in the mean flow relative to that seen in weak-to-moderate shear cases.

Given the different tilting mechanisms described above, it is not entirely clear to us which are most important under relatively weak-shear environments and to what extent they contribute to the long-term organization of MCVs. Most notably, for the case of relatively weak shear, DW94 and SWK offer differing conclusions. The studies of DW94 and Weisman and Davis (1998), moreover, suggest that tilting of horizontal vorticity is the primary mechanism in enhancing and organizing vertical vorticity into mesoscale circulations during both the early and mature stages of the storm. Although DW94 state that convergence "eventually", becomes important (DW94, p. 2020), they do not go further to investigate the role it plays in the amplification and organization of vertical vorticity. It is therefore unclear in their study when, and to what degree, vorticity convergence becomes a significant contributor to the vorticity amplification. While we agree that tilting of both negative and positive horizontal vorticity are essential in generating the initial vertical vorticity, we will show that vorticity convergence plays an equally important role in organizing and amplifying the circulations during the early to mature stages of the storm.

This conclusion is supported by a Lagrangian vorticity budget analysis using backward trajectories as a method of determining the processes by which air parcels acquire vertical vorticity. Weisman and Davis (1998) used backward trajectories to show that parcels located along vortex lines associated with storm-generated negative horizontal vorticity originate from the low-level ambient flow ahead of the system and also from the rear of the system. These air parcels were carried upward by the front-to-rear inflow and descended from cooler levels above via the rear inflow current, respectively. While this establishes the origin of air parcels along these vortex lines, a Lagrangian vorticity analysis of the tendency terms in the vertical vorticity equation following the trajectories was not carried out.

Trier et al. (1997) analyzed Lagrangian backward trajectories as a way of establishing the source of vertical vorticity in simulations of a large tropical squall line observed during Tropical Ocean and Global Atmosphere Coupled Ocean-Atmosphere Response Experiment 
(TOGA COARE). By calculating a vertical vorticity budget along the trajectories, they showed that the primary generator of the initial vertical vorticity of the air parcels was the tilting of storm-generated horizontal vorticity within the interface formed by the cold pool and leading-line updrafts, while tilting of ambient horizontal vorticity was found to be less effective. Once the air parcels acquired their initial vertical vorticity, vorticity convergence was the dominant vertical vorticity tendency term. The Lagrangian analysis provided in this study is similar in design to that of Trier et al. (1997), but the differences in the initial conditions between their tropical analysis and the present midlatitude analysis do not allow one to assume a priori that similar results will be achieved. It is worthwhile, therefore, to carry out a Lagrangian analysis in the present study so as to provide a midlatitude comparison to its tropical counterpart.

The objective of the present study then is to further investigate the processes responsible for the generation and subsequent organization of MCVs in an idealized, finite-length convective line within midlatitude environmental conditions. We will base our experiment on that carried out by DW94, which consists of a finitelength convective line in a relatively weak, westerly shear environment. Following DW94, we neglect Coriolis effects, for reasons cited therein. The plan of this paper is as follows. Section 2 gives an overview of the numerical experiment and compares PV fields with previous studies. A discussion of vertical vorticity formation and evolution of the convective line during the first $3 \mathrm{~h}$ of the simulation follows in section 3 . Here we examine the behavior of vertical velocity as well as the role of vorticity convergence and tilting tendencies in vertical vorticity generation. The aforementioned Lagrangian trajectories and corresponding trajectory budget analyses are presented in section 4 . We conclude in section 5 with a summary of our principal findings.

\section{Numerical experiment and simulation overview}

The Regional Atmospheric Modeling System (RAMS), a nonhydrostatic, primitive-equation model that has proven useful in regional and mesoscale studies, was used for the simulation analyzed here. Details regarding model equations, vertical coordinate, grid structure, time differencing, and available parameterizations (e.g., turbulence) can be found in Pielke et al. (1992).

The model configuration and thermodynamic sounding for model initialization were identical to the weakshear simulation of DW94, which includes a shear profile consisting of easterly winds of $-10 \mathrm{~m} \mathrm{~s}^{-1}$ near the surface, increasing linearly to zero at $z=2.5 \mathrm{~km}$ and constant winds above. The discretization is on a single grid consisting of $150 \times 150$ horizontal grid points at 4-km spacing, and 30 vertical levels with constant 700$\mathrm{m}$ spacing. No surface fluxes or radiation parameterization were employed; a free-slip lower boundary con- dition is imposed. The turbulence parameterization uses the Mellor and Yamada (1974) scheme to compute vertical mixing coefficients, while a local deformation scheme (Smagorinsky 1963) is used for horizontal mixing. A grid-scale microphysics parameterization (Walko et al. 1995) with cloud water and rain processes is used. Ice processes are not included. Velocity components and thermodynamic data in the model are defined on a Mesinger and Arakawa (1976) staggered C grid, and in the processing of model output, winds are interpolated to the thermodynamic grid points prior to differentiations needed in postprocessing. Six warm bubbles, symmetrically spaced with the same strength as DW94 and geometry described in Klemp and Wilhelmson (1978), initiated the convective line. The simulation was then run for $6 \mathrm{~h}$. The east-west line located at $y=300 \mathrm{~km}$ dividing the horizontal domain into equally sized north and south sectors will be hereafter referred to as the "centerline."

The weak-shear simulation has become the most extensively examined case in past model studies of midlatitude squall lines of finite extent (e.g., DW94, SWK, Weisman and Davis 1998) and appears to have become a benchmark case upon which most finite-extent squall line simulations are based. In spite of this, recent studies examining the weak-shear case offer differing conclusions as to what role the dynamic processes of vorticity tilting and convergence play in the development and organization of MCVs (DW94, SWK, Weisman and Davis 1998), thus suggesting this case is still not fully understood and requires further attention. It is for this reason we choose to focus our simulation solely on this important weak-shear case.

An exact comparison of detailed results between our simulation and that of DW94 was not anticipated due to differences in model numerics and microphysical parameterizations. Our results nevertheless confirm that the main features of DW94 were reproduced. It is unlikely that two nonhydrostatic, primitive-equation models with identical initial conditions would produce similar results (e.g., in the vertical vorticity field) due to entirely different physical mechanisms. Were this the case, the methodology of using numerical models for this type of investigation would be in question.

Figure 1a shows PV at $z=2.1 \mathrm{~km}$ superimposed on vectors of horizontal wind perturbations at $3 \mathrm{~h}$, where the PV $(q)$ is given by

$$
q=\frac{\omega \cdot \nabla \theta}{\rho} .
$$

Here, $\omega$ is the absolute vorticity vector, $\theta$ is potential temperature, and $\rho$ is density of dry air. An arithmetic average of the PV and velocity fields between the model levels at $z=1.75 \mathrm{~km}$ and $z=2.45 \mathrm{~km}$ is calculated to display the data at $z=2.1 \mathrm{~km}$, the level displayed by DW94. The data is also coarsened to a $20-\mathrm{km}$ grid, as done in DW94. Although this can give misleading im- 
$3 \mathrm{~h}$

(a)

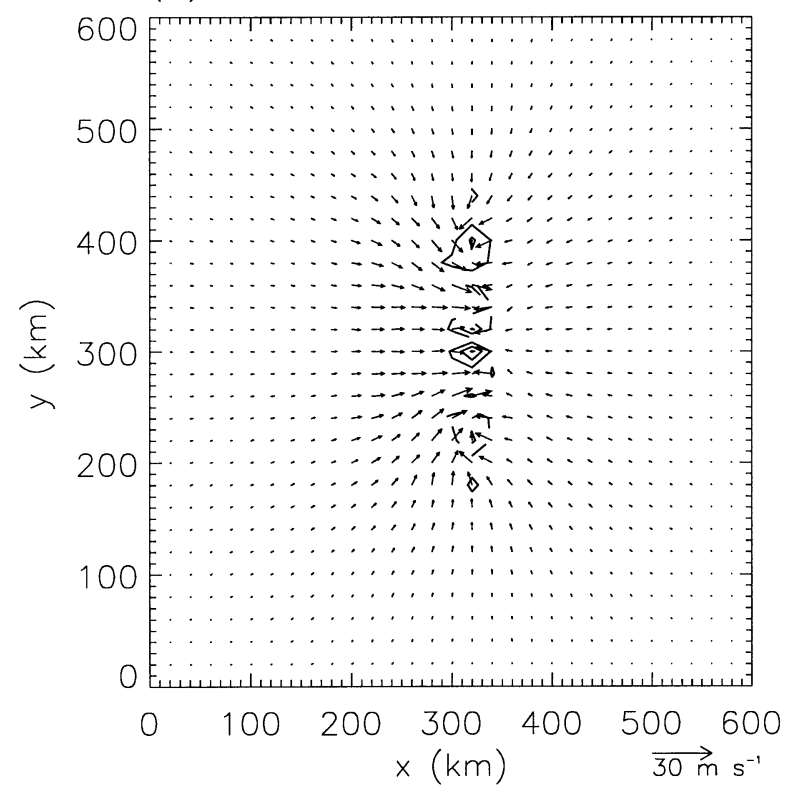

(c)

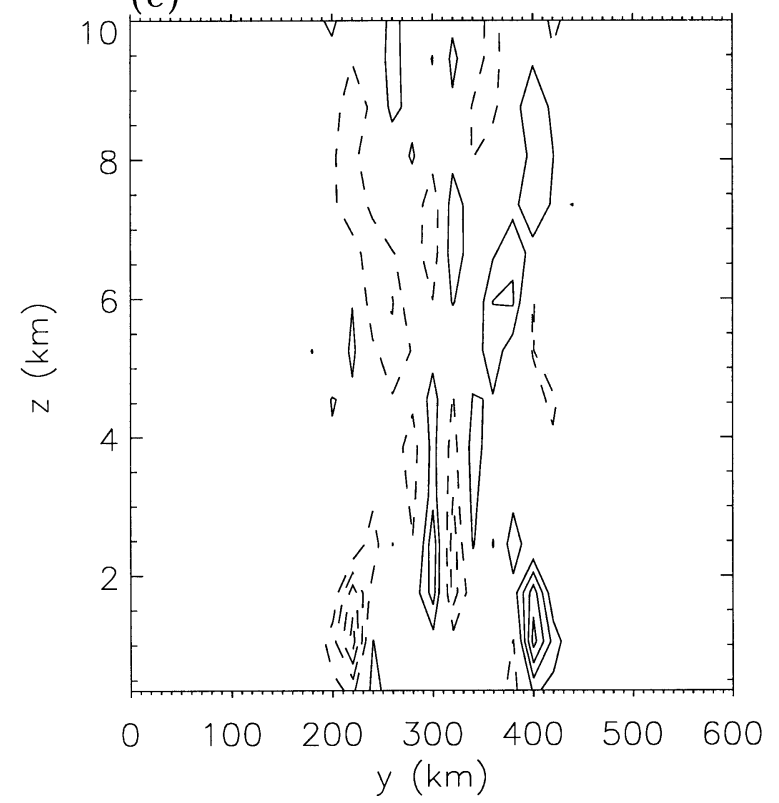

$6 \mathrm{~h}$

(b)

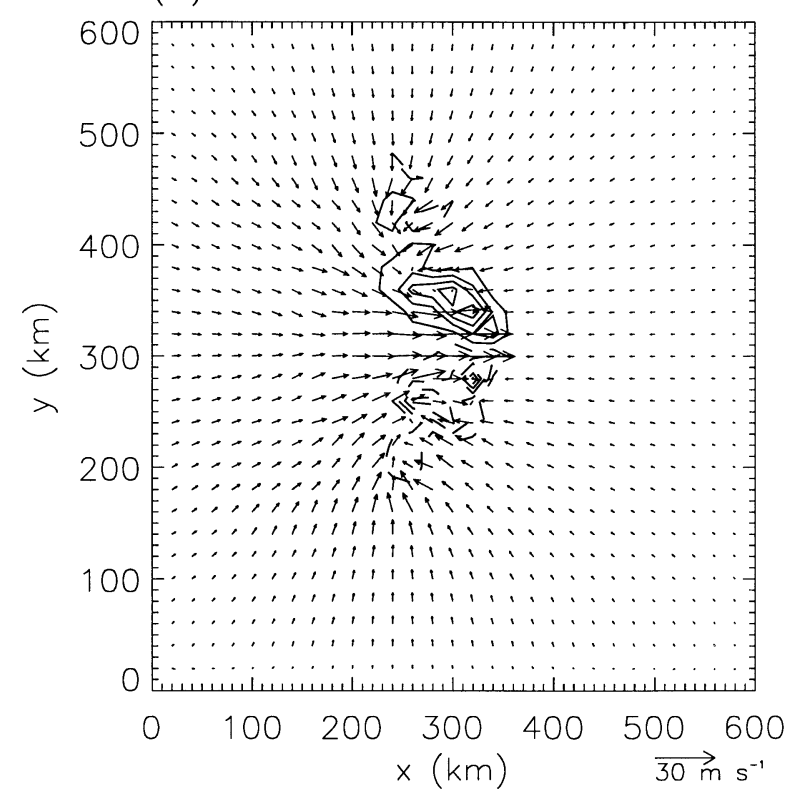

(d)

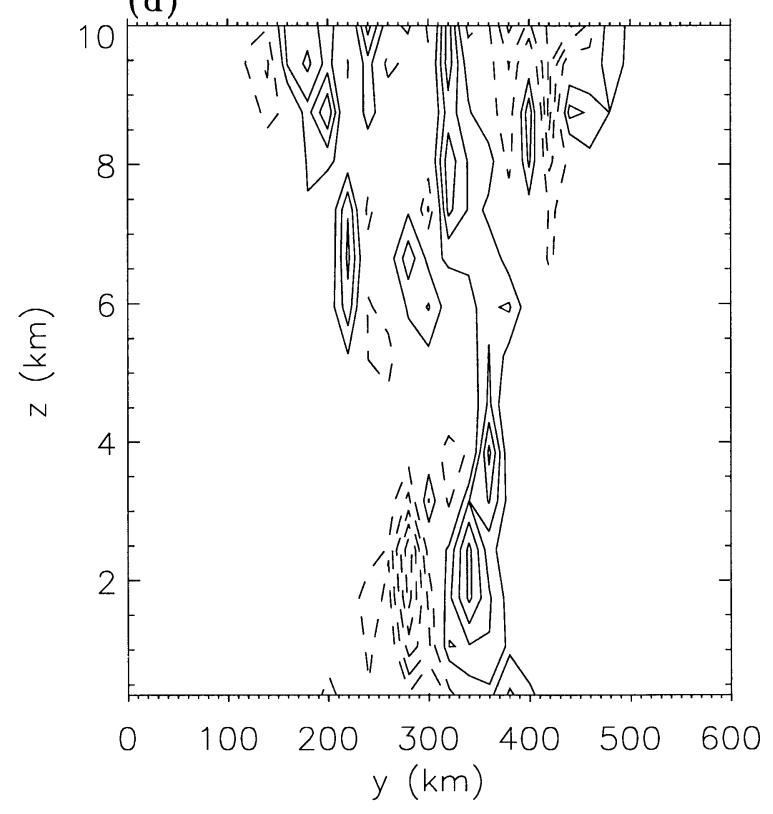

FIG. 1. PV and horizontal velocity perturbation at $z=2.1 \mathrm{~km}$ at (a) $3 \mathrm{~h}$ and (b) $6 \mathrm{~h}$. North-south vertical cross sections of PV at $x=$ $320 \mathrm{~km}$ at (c) $3 \mathrm{~h}$ and (d) $6 \mathrm{~h}$. Contour interval for PV is $1.0 \mathrm{PVU}\left(1 \mathrm{PVU}=10^{-6} \mathrm{~K} \mathrm{~m}^{2} \mathrm{~kg}^{-1} \mathrm{~s}^{-1}\right)$. Positive values are represented by solid contours and negative values by dashed contours for this and all other figures unless otherwise noted. The zero contour has been omitted.

pressions regarding the detailed distribution of the actual fields, it is adequate for highlighting the mesoscale features of the data. Already evident in Fig. 1a are regions of positive and negative PV at the north and south ends of the line, respectively. By this time, the wind vectors already exhibit cyclonic flow perturbations in the northern sector and anticyclonic flow perturbations in the southern sector of the convective line, with westerly wind anomalies approaching $10 \mathrm{~m} \mathrm{~s}^{-1}$ between the two main end vortices. A north-south section through the coarsened PV field at $3 \mathrm{~h}$ (Fig. 1c) shows a positive low-level PV maximum to the north with negative PV south. Weaker areas of PV of both signs are seen aloft.

By $6 \mathrm{~h}$, the PV distribution displays even more welldefined regions of positive $\mathrm{PV}$ to the north and negative PV to the south of the centerline (Fig. 1b). The size and 
magnitude of PV minima and maxima have increased, indicating that system-scale dynamical processes are at work, which are organizing the original positive and negative regions. The convective line has become more "bow" shaped, rear inflow has increased and the line center has surged forward. From a north-south section through the coarsened field of PV (Fig. 1d) we see a more well-defined and vertically deeper PV dipole than at $3 \mathrm{~h}$. Evident in both the horizontal and vertical crosssectional plots in Fig. 1 is the apparent movement of the cyclonic and anticyclonic vortices at the line-ends toward each other between 3 and $6 \mathrm{~h}$. By $6 \mathrm{~h}$, the centers of the main PV anomalies approach to within $100 \mathrm{~km}$ of each other, also consistent with DW94. The magnitude of the PV anomalies at the northern and southern line-ends of the convective line at $3 \mathrm{~h}$ is 1.0 PVU (1 $\left.\mathrm{PVU}=10^{-6} \mathrm{~m}^{2} \mathrm{~K} \mathrm{~kg}^{-1} \mathrm{~s}^{-1}\right)$. By $6 \mathrm{~h}$, the PV magnitudes increase to 4.0 PVU. These are approximately the same values shown in DW94 ${ }^{1}$. Westerly wind perturbations between the line ends approach 10 and $20 \mathrm{~m} \mathrm{~s}^{-1}$ at 3 and $6 \mathrm{~h}$, respectively, also comparable to that observed in DW94. The dipolar structure in the PV field and the trend in the PV field between 3 and $6 \mathrm{~h}$ compares well with DW94, with increasing and decreasing PV at the northern and southern line ends, respectively.

\section{Vorticity evolution and formation mechanisms}

Previous studies have focused primarily on vertical vorticity formation mechanisms from 3 to $6 \mathrm{~h}$ after a line of warm bubbles triggers convective-line formation. But as is evident from the previous section, there already exist cyclonic and anticyclonic circulations with a size on the order of $100 \mathrm{~km}$ by $3 \mathrm{~h}$ into the present simulation. In this section we address four specific questions. How early is vertical vorticity seen, and what is responsible for its formation? Since the convective line is initiated by a symmetric line of warm bubbles, when does the asymmetry in the vertical vorticity field appear and how does it form? The most coherent vortices were observed at $z=1.05 \mathrm{~km}$ and $z=1.75 \mathrm{~km}$ in the model, thus we will concentrate our analysis on the formation of vertical vorticity at these two levels.

Figure 2 shows a time series of the average vertical vorticity over the north (solid line) and south (dashed line) half of the model domain over the first $3 \mathrm{~h}$ at $z=$ $1.05 \mathrm{~km}$. For the first $1.5 \mathrm{~h}$, the average vertical vorticity in both halves remains small. As we will see, this is not due to very weak vertical vorticity production. Rather, almost equal regions of positive and negative vertical vorticity in each half of the domain cancel. A dramatic change occurs shortly after $1.5 \mathrm{~h}$ as an obvious asymmetry forms with cyclonic vorticity north and anticyclonic vorticity south. Absolute values of average vorticity increase by a factor of approximately 7 over 30

\footnotetext{
${ }^{1}$ The 0.5 PVU contour interval indicated in Fig. 2 of DW94 should be corrected to 1.0 PVU (C. A. Davis 2001, personal communication).
}

min, which demonstrates that an asymmetry forms rapidly in the very early stages of MCS organization. This asymmetry, or vorticity dipole, is in the same sense as in simulations by DW94, SWK, and Weisman and Davis (1998) and is likely due to the amalgamation of the initially separate cold pools associated with each individual convective cell.

The evolution of vertical velocity, vertical vorticity, and vertical vorticity tendencies during the first $3 \mathrm{~h}$ of the MCS lifecycle have not been presented in similar previous studies. Since these are of importance to the MCV evolution, we explore the early stages of the MCS here. Shown in Fig. 3 are vertical velocity and horizontal wind vectors at $z=1.05 \mathrm{~km}$ every $0.5 \mathrm{~h}$ over the first 3 h. Figures 4, 5, and 6 show the corresponding fields of vertical vorticity, vorticity tilting, and convergence tendencies, as defined in the equation for the material rate of change of vertical vorticity, given by

$$
\begin{aligned}
& \frac{D \zeta}{D t}=-\zeta\left(\frac{\partial u}{\partial x}+\frac{\partial v}{\partial y}\right)-\left(\frac{\partial w}{\partial x} \frac{\partial v}{\partial z}-\frac{\partial w}{\partial y} \frac{\partial u}{\partial z}\right) \\
&+\underbrace{\frac{1}{\rho^{2}}\left(\frac{\partial \rho}{\partial x} \frac{\partial p}{\partial y}-\frac{\partial \rho}{\partial y} \frac{\partial p}{\partial x}\right)}_{\text {convergence }}+\left.\right|_{\text {tilting }} ^{\mid \frac{\partial F_{y}}{\partial x}-\frac{\partial F_{x}}{\partial y}} . \\
& \mid \begin{array}{c}
\mid \text { turbulent fluxes } \\
\mid
\end{array}
\end{aligned}
$$

Here, $\zeta$ is the vertical vorticity, and the terms on the right-hand side of the equation (reading from left to right) represent vorticity convergence (stretching), tilting, solenoidal effects, and subgrid-scale parameterization. Only the results at $z=1.05 \mathrm{~km}$ are shown and discussed in this section, since the winds, vertical vorticity, and tendency fields at $z=1.75 \mathrm{~km}$ are similar. The data shown in these figures have not been coarsened to a $20-\mathrm{km}$ horizontal grid as was done in section 2 .

At $0.5 \mathrm{~h}$ (Fig. 3a) six convective updrafts from the six warm bubbles are observed. Each updraft generates an associated vertical vorticity dipole (Fig. 4a). Most of the vertical vorticity appears to be due to tilting of ambient shear by each updraft, which is simply vorticity tilting by single convective cells as described by Klemp and Wilhelmson (1978). Vorticity convergence adds to the vertical vorticity pattern and is stronger (Fig. 6a), but spatially less extensive than tilting (Fig. 5a).

By $1 \mathrm{~h}$, the initial line of convective updrafts collapses and is replaced by a line of downdrafts. Vertical vorticity formed by tilting of the initial updrafts has been advected westward. New vertical vorticity forms as a result of the ambient shear being tilted by the downdrafts, and the regions of positive-negative tilting have reversed in comparison to $0.5 \mathrm{~h}$. This reversal can been seen in the vertical vorticity field, as the positive-negative vertical vorticity pattern has reversed in association with each convective cell. Vorticity convergence is much weaker than tilting at this time.

Between 1 and $2 \mathrm{~h}$, cold outflow associated with ini- 


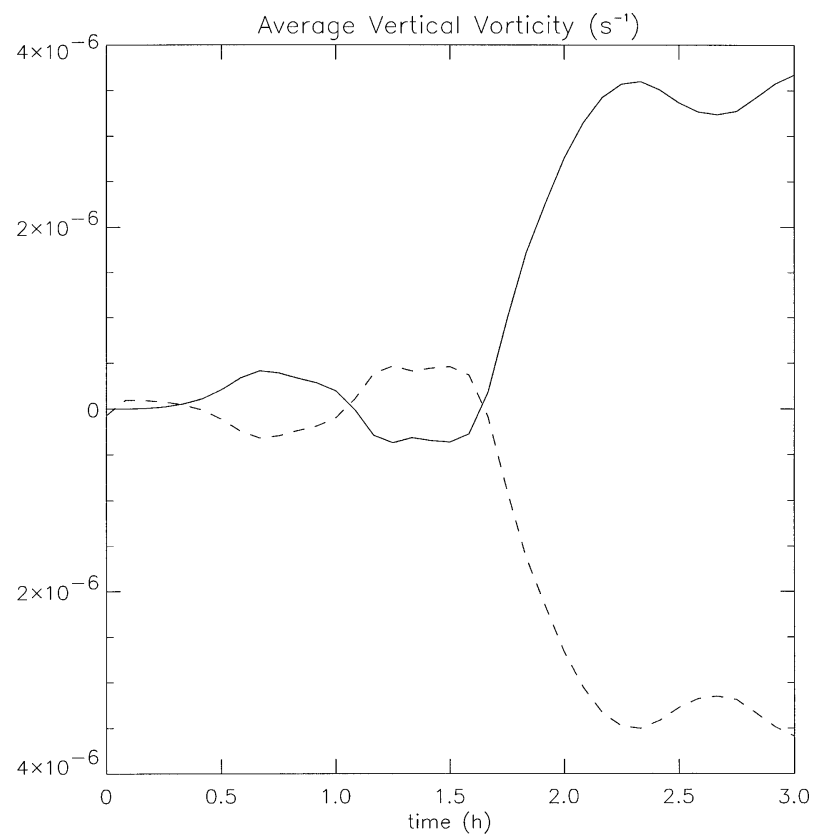

FIG. 2. Vertical vorticity averaged horizontally over the model domain north and south of the centerline at $z=1.05 \mathrm{~km}$. The solid line is average vertical vorticity north of the centerline, and the dashed line is the average vertical vorticity south of the centerline.

tial convective cell downdrafts and precipitation has triggered new convection. At $1.5 \mathrm{~h}$, the strongest convective updrafts form between previous downdrafts, while weaker cells form along the forward-spreading cold outflow. The vertical vorticity pattern becomes more complicated, consistent with increasingly complex fields in both vorticity tilting and convergence tendencies. The wind fields, however, show signs of organization as represented by cyclonic and anticyclonic flow at the northern and southern line ends, respectively.

By $2 \mathrm{~h}$, the leading convective line has become more organized with downdrafts to the rear, and vertical vorticity is spatially more extensive. The asymmetry implied by Fig. 2 is now visibly apparent in the vertical vorticity field as well, though strong areas of positive and negative vertical vorticity are still found between the line-end vortices. Tilting is still the dominant vertical vorticity formation mechanism, but a clear asymmetry with more positive tilting tendencies in the northern sector and negative tilting tendencies in the southern sector is not evident. Figure 7 shows vertical velocity, wind vectors and zonal shear averaged over the north half of the convective line at $2 \mathrm{~h}$. Tilting of easterly zonal shear (associated with the cold pool), as proposed by Weisman and Davis (1998), is seen at this time, but it is very localized to a horizontal spatial scale of ap- proximately $5 \mathrm{~km}$ in the east-west direction. By comparison, although vorticity convergence is generally weaker and less extensive than tilting, it does exhibit a clear asymmetry, with positive to the north and negative to the south (Fig. 6d), and has a horizontal scale commensurate with the vertical vorticity anomalies. Thus while it appears that tilting of convectively produced horizontal vorticity leads to the initial asymmetry in vertical vorticity, vorticity convergence enhances and organizes the vorticity produced via tilting. This view stands in contrast to the conclusions given in DW94 and Weisman and Davis (1998), which imply that vorticity convergence is secondary to tilting as it relates to enhancing and organizing vertical vorticity at these early times.

Between 2 and $3 \mathrm{~h}$, the convective line surges forward; the vertical vorticity dipole now appears to be causing local enhancement of rear-inflow (Weisman and Davis 1998), which deforms the convective line at both the north and south ends. Positive and negative regions of vertical vorticity continue to expand on the north and south ends of the convective line, respectively, which appears to be due more to vorticity convergence. Tilting of both signs occurs throughout the convective line, but tilting is mostly negative at the north end and is mostly positive at the south end. At first glance this appears to be contradictory to what is observed in vertical vorticity. The Eulerian figures shown here, however, do not reveal what is occurring at vertical levels other than the level displayed. Since only tilting can produce the asymmetry in vertical vorticity (neglecting solenoidal effects and friction), air parcels acquire the sign of vorticity via tilting that is consistent with the vertical vorticity asymmetry at vertical levels above and below the level displayed. Convergence then stretches the vorticity of the air parcels at the level displayed in Figs. 3-6. Therefore, contrary to what is implied in DW94 and Weisman and Davis (1998) for their weak-shear simulations, our findings suggest that for the moderate range of shears considered, tilting and vorticity convergence are both equally important factors in organizing vertical vorticity into MCVs. Although vorticity convergence is weak during the first hour of the simulation, it becomes significant by $3 \mathrm{~h}$-which is still early in the MCS lifecycle-as the updrafts become more organized. These conclusions will be further clarified in the Lagrangian trajectory analysis discussed in the next section.

\section{Lagrangian analysis}

The Eulerian analysis discussed thus far is helpful as a first step in diagnosing some aspects of the dynamics of the convective line. However, since Eulerian plots

FIG. 3. Vertical velocity contours and horizontal wind vectors at $z=1.05 \mathrm{~km}$ at (a) $0.5 \mathrm{~h}$, (b) $1.0 \mathrm{~h}$, (c) $1.5 \mathrm{~h}$, (d) $2.0 \mathrm{~h}$, (e) $2.5 \mathrm{~h}$, and (f) $3.0 \mathrm{~h}$. The contour interval for vertical velocity is $1.0 \mathrm{~m} \mathrm{~s}^{-1}$ and the zero contour has been omitted. Wind vectors are system-relative. 

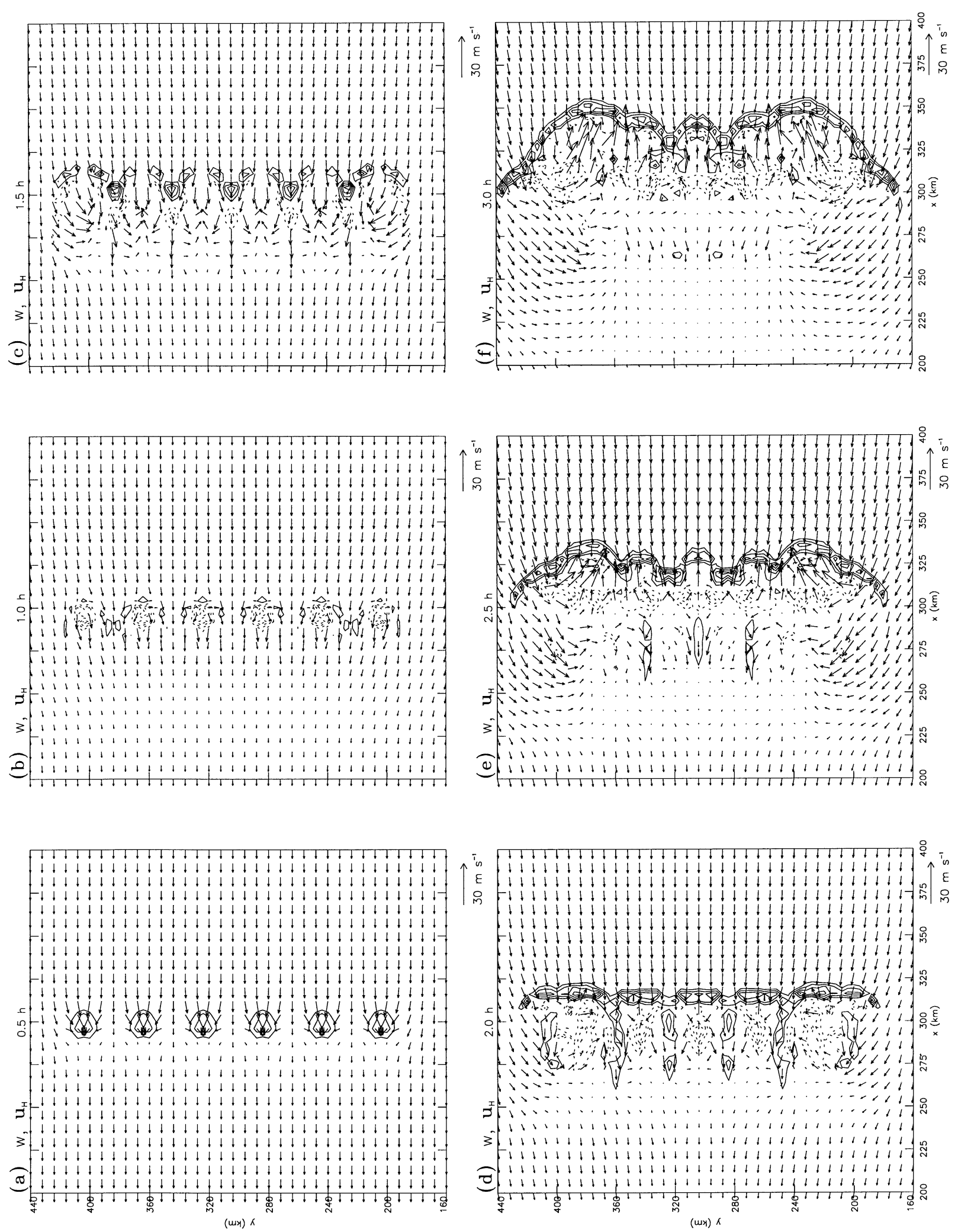

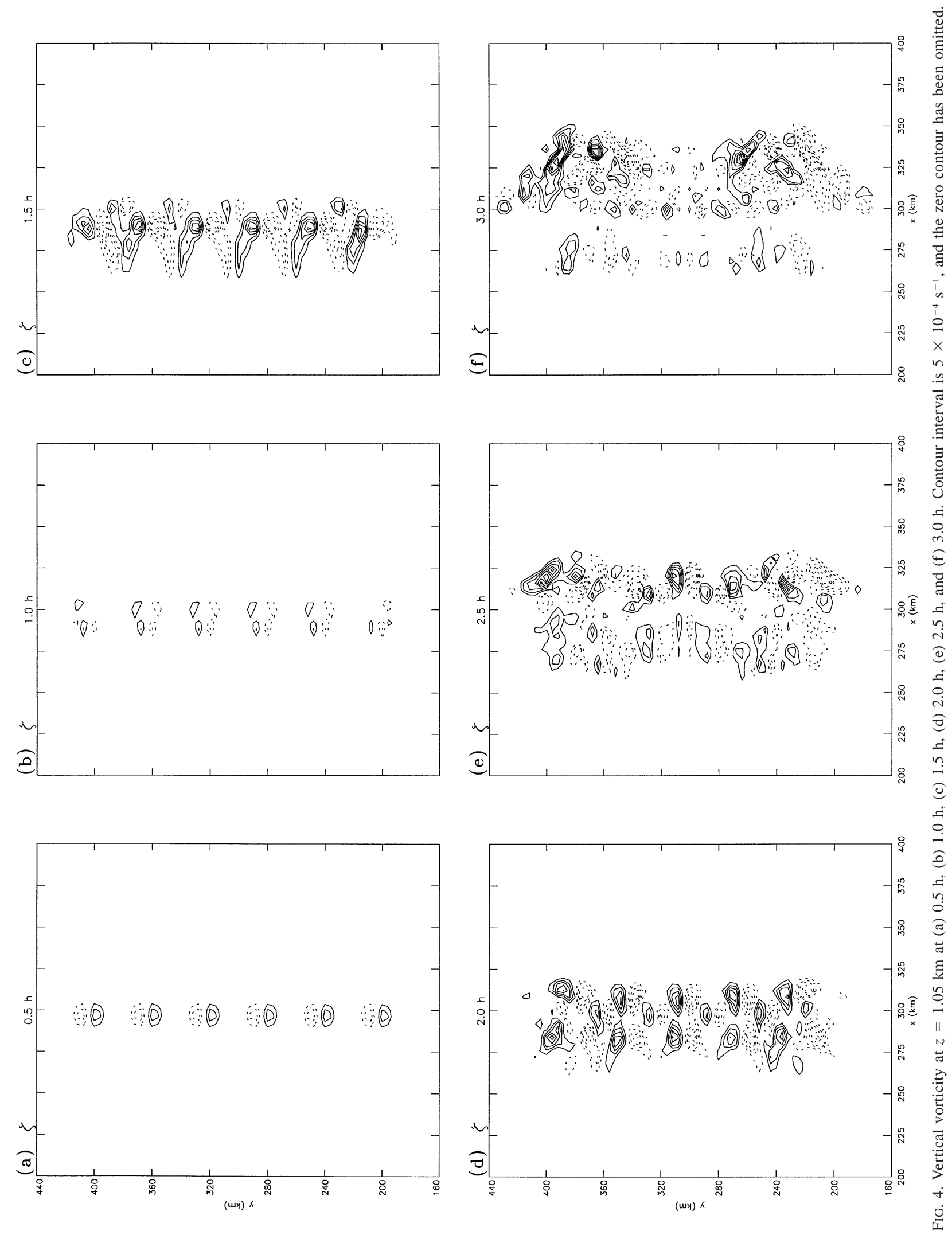

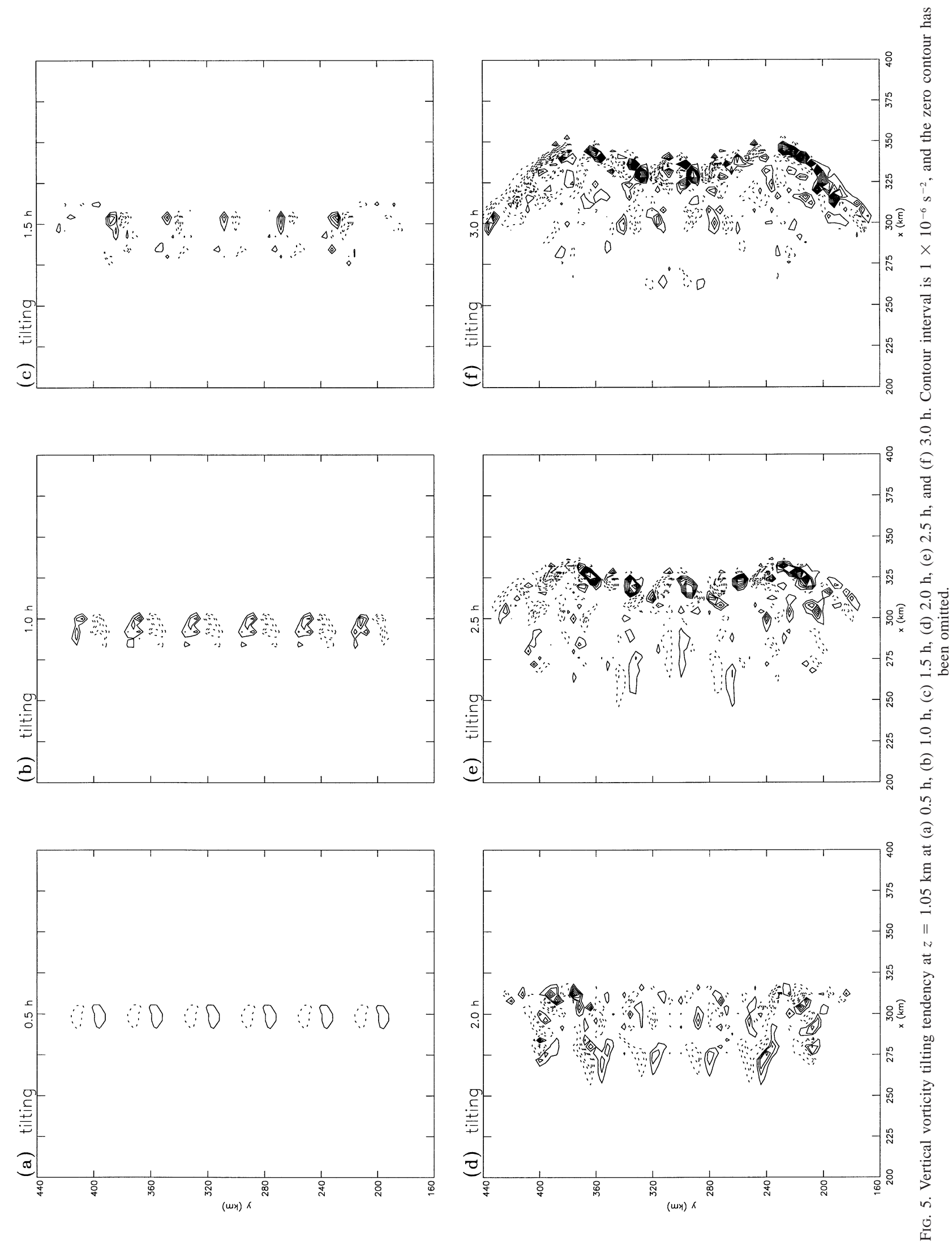

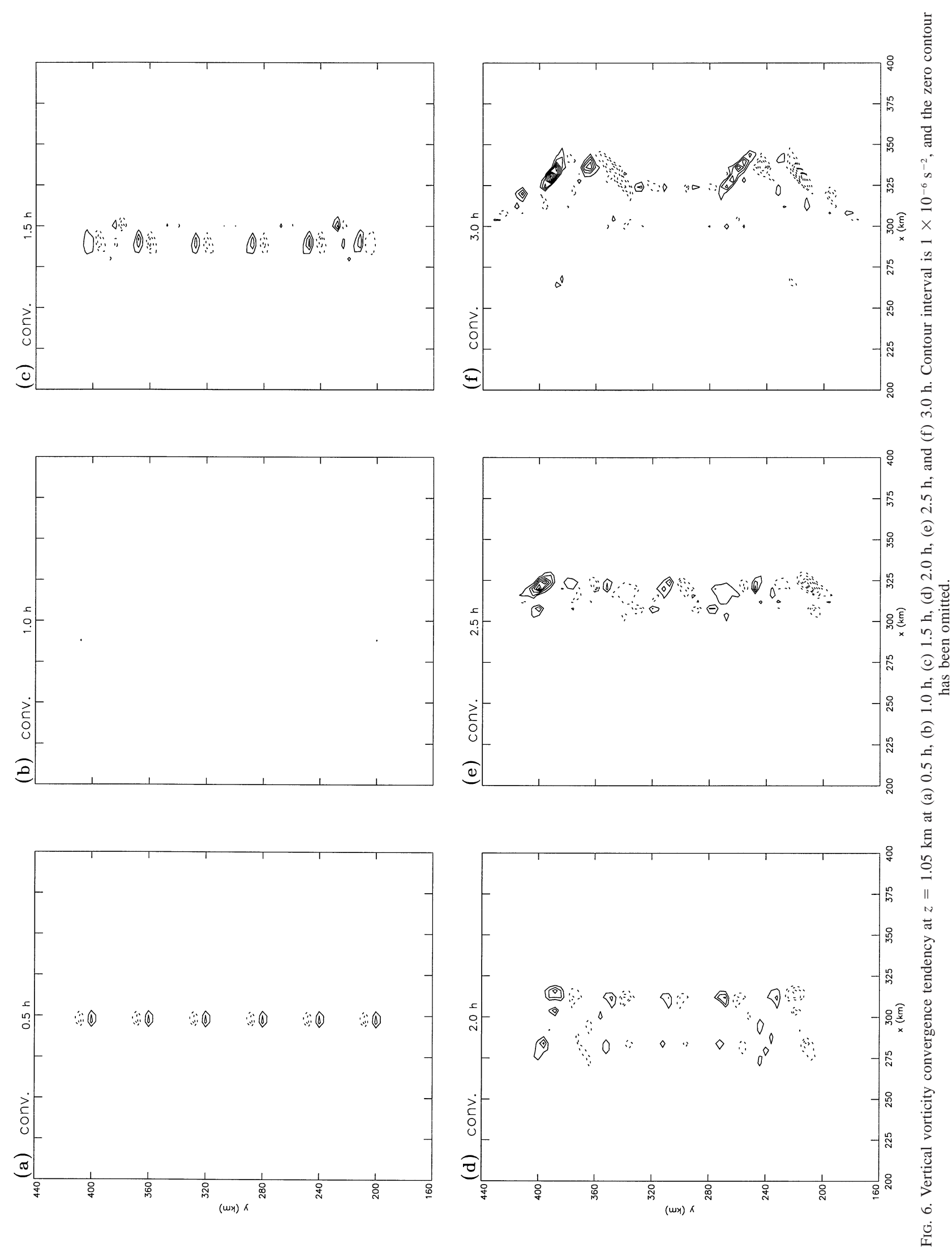


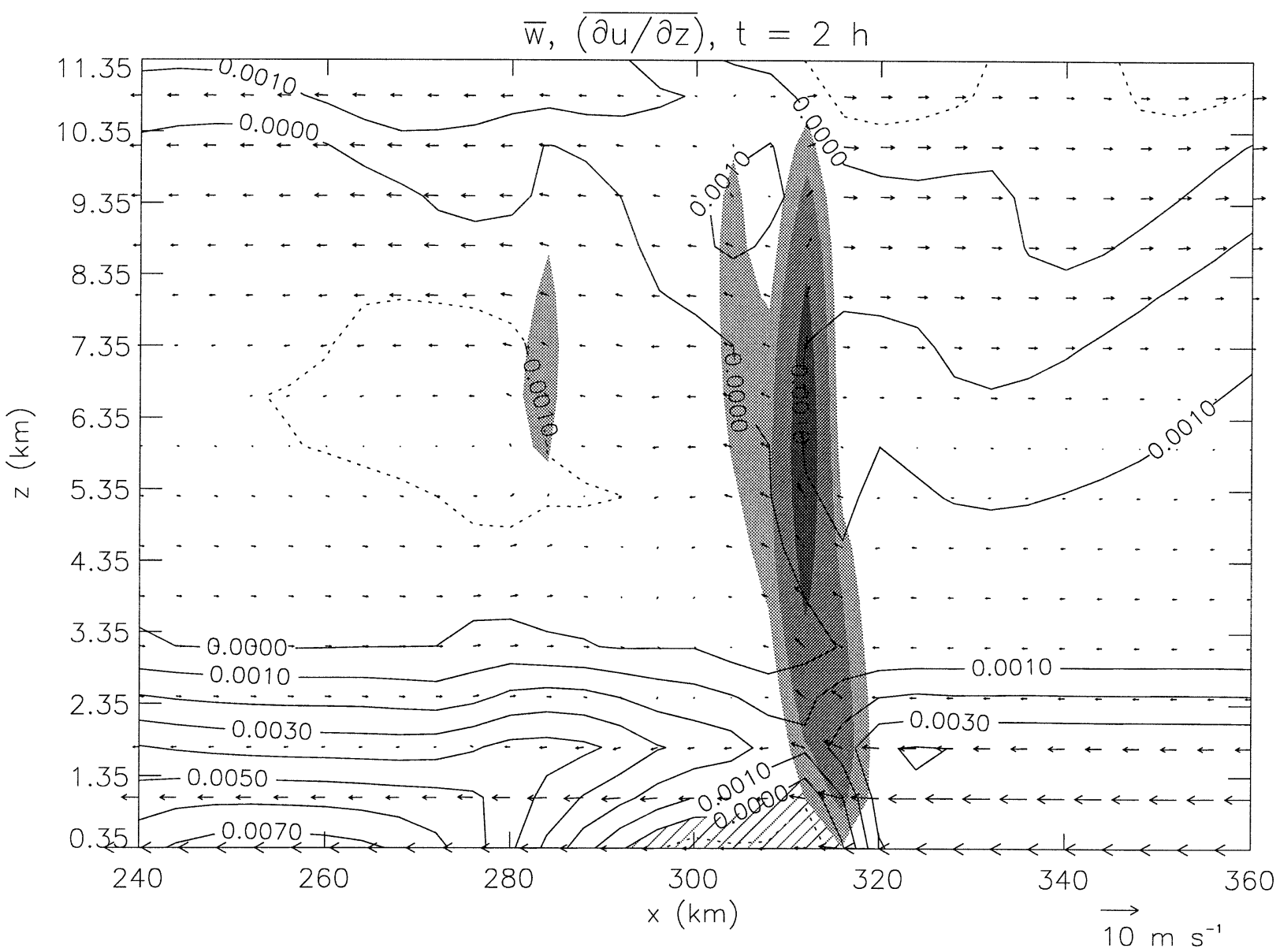

FIG. 7. East-west vertical cross section of system-relative flow, zonal shear, and vertical velocity averaged horizontally north of the centerline. Zonal shear (contoured) is plotted at intervals of $10^{-3} \mathrm{~s}^{-1}$. Shading represents magnitudes of average vertical velocity greater than $0.5 \mathrm{~m} \mathrm{~s}^{-1}$ (light shading), $1.0 \mathrm{~m} \mathrm{~s}^{-1}$ (medium shading), and $2.0 \mathrm{~m} \mathrm{~s}^{-1}$ (dark shading). Cross-hatching represents negative horizontal vorticity generated by the gust front at low levels.

only show "snapshots," it may be misleading and at a minimum does not furnish a complete picture of the evolution of the squall line. To further our understanding, a Lagrangian particle trajectory analysis focusing on the significant terms in the vertical vorticity equation (2) is carried out. Time-dependent backward trajectories originating near the center of maximum vertical vorticity are analyzed at $z=1.05,1.75$, and $2.45 \mathrm{~km}$, and all terms in the vertical vorticity equation are calculated for parcels along their trajectories. In addition, the tilting and vorticity convergence tendency terms are integrated in time along the trajectories in order to measure their respective contribution to vertical vorticity production at any point on the trajectory. Winds at 5-min intervals are used and the trajectories are calculated on the 4-km horizontal grid using a four-dimensional (space-time) linear interpolation scheme with a temporal increment of $30 \mathrm{~s}$.

Although the analysis provided here is similar to that done by Trier et al. (1997), the initial conditions in the present midlatitude simulation vary considerably from their tropical case. Specifically, the thermodynamic profile in the present case has more CAPE $\left(\sim 2400 \mathrm{~J} \mathrm{~kg}^{-1}\right.$ versus $\sim 1500 \mathrm{~J} \mathrm{~kg}^{-1}$ ) and the vertical shear environment has approximately twice the ambient horizontal vorticity of Trier et al. (1997; the unidirectional shear inferred from their hodograph is approximately $5 \mathrm{~m} \mathrm{~s}^{-1}$ over the lowest $3 \mathrm{~km}$ ). Moreover, the shear profile used in the present study is both unidirectional and linear with height whereas Trier et al. (1997) implemented a curved hodograph that is nonlinear with height. Given the nonlinearity of the mesoscale dynamics investigated, it is not obvious that such differences in initial conditions will produce similar behavior in terms of vorticity tilting and vorticity convergence. We are unaware of any other midlatitude squall line study that investigates the role of tilting versus vorticity convergence in the early evolution of the squall line and therefore consider it useful to carry out the present Lagrangian analysis.

Ideally, the sum of integrated convergence and tilting tendencies should approximately equal the instantaneous vertical vorticity at any one time. This is ap- 
proximately true in our case, although it should be noted that interpolation and discretization errors resulting from the trajectory calculations account for discrepancies seen in the budget analyses. Accurate calculation of integrated terms along interpolated trajectories such as these can be difficult since calculation errors will accumulate in the integral summation. Over 100 trajectories were calculated at each level in order to check consistency, and we are confident the budget analyses represent the model's contribution of the convergence and tilting tendencies. ${ }^{2}$

Vertical vorticity generation due to solenoidal effects and subgrid-scale turbulence parameterization was found to be at least an order of magnitude smaller than the vorticity convergence and tilting tendencies, thus these contributions will not be discussed. Since trajectories at all three vertical levels mentioned produce similar results, we present here only those originating at $z=1.75 \mathrm{~km}$. The trajectory and vorticity budget results from the southern anticyclonic vortex region mirror those from the northern vortex region, thus only the trajectory analyses from the latter are presented here. Presented in this section is one backward trajectory from $3 \mathrm{~h}$, which is typical of backward trajectories originating from the vertical vorticity maximum at the vertical level shown.

Figure 8a shows a three-dimensional view of a backward trajectory from $3 \mathrm{~h}$ originating at $z=1.75 \mathrm{~km}, x$ $=324 \mathrm{~km}$, and $y=392 \mathrm{~km}$, which is near the center of an area of large vertical vorticity at $z=1.75 \mathrm{~km}$ and $t=3 \mathrm{~h}$. Figure $8 \mathrm{~b}$ shows the same trajectory as viewed in the horizontal plane superimposed on vertical vorticity contours at $z=1.75 \mathrm{~km}$ and $t=3 \mathrm{~h}$. The trajectory was followed back $3 \mathrm{~h}$ to the start of the simulation and time intervals of $30 \mathrm{~min}$ are marked on the trajectory by solid dots. The Lagrangian particle is lifted in the updraft at $2 \mathrm{~h}$, roughly the same time it starts a cyclonic turn into the developing circulation. The parcel is lifted up to $z=3 \mathrm{~km}$, at which point it is brought back down to $z=1.75 \mathrm{~km}$ by downdrafts.

Figure $8 \mathrm{c}$ shows the instantaneous vertical vorticity and the instantaneous convergence and tilting tendency terms following the trajectory; Fig. $8 \mathrm{~d}$ shows the tendency terms integrated along the trajectory. Shortly before the air parcel encounters the updraft region at 120 min it acquires a small amount of negative vertical vor-

\footnotetext{
${ }^{2}$ Analysis of Lagrangian trajectories during the post-3-h period produced inconsistent results and showed sensitivity to initial conditions, thus suggesting that a Lagrangian trajectory analysis performed at the current spatial and temporal resolution during the mature stages of a squall line type system is problematic. This sensitivity is believed to be due to discretization errors in association with embedded convection in the system through which air parcels travel and is likely the reason DW94 did not carry out a similar Lagrangian analysis at these later times (DW94, p. 2019). In contrast, Trier et al. (1997) performed a Lagrangian analysis at $4 \mathrm{~h}$ in which the instantaneous vertical vorticity values were consistent with the integrated tendency terms along their trajectories. We believe they were successful because the nested-grid model they employed resolved regions of active convection to horizontal grid spacings of $1 \mathrm{~km}$.
}

ticity, but then enters the developing region of cyclonic circulation and the diagnosed value of vertical vorticity increases steadily up to $1.0 \times 10^{-3} \mathrm{~s}^{-1}$ at $140 \mathrm{~min}$. The diagnosed tilting tendency is slightly positive during this time but decreases and becomes negative after $140 \mathrm{~min}$. This decrease is associated with a brief decrease in the diagnosed vertical vorticity. Vorticity convergence then becomes the dominant tendency term, which corresponds to a steady increase in vertical vorticity until approximately $155 \mathrm{~min}$. The tilting tendency remains mostly negative up to $3 \mathrm{~h}$, which contributes to a negative tendency in vertical vorticity. This is consistent with the Eulerian results shown in Figs. 5 and 6. A look at the integrated tendency terms in Fig. 8d reveals that the tilting tendency is the main contributor to vertical vorticity production in the air parcel up to approximately $140 \mathrm{~min}$. At this time, the integrated tilting decreases and the integrated convergence dominates the enhancement of positive vertical vorticity until shortly before $3 \mathrm{~h}$.

Tilting plays an essential role in the early stages as the initial asymmetry forms, but convergence becomes a comparable, and at times dominant, tendency term at $2.5 \mathrm{~h}$ and later. The mixed role that tilting and vorticity convergence assume in organizing vertical vorticity is in agreement with Trier et al. (1997, cf. their Figs. 20 and 21), although this change progresses at a slower pace in their simulation. It is believed that this is in part due to the different initial conditions in their simulation.

When applying the circulation budget given in Weisman and Davis [1998; Eqs. (4)-(6)] over the entire northern half of the system, it is apparent that the vorticity convergence term does not contribute to the rate of change of the average vertical vorticity. Their circulation budget shows that the source of mesoscale vortices is due to tilting, but does not explain how the mesoscale vortices are constructed. In a global sense, localized increases in vertical vorticity owing to horizontal convergence are being compensated by localized decreases in vertical vorticity due to horizontal divergence. One must therefore analyze subregions of the domain to discover how local maxima in the vorticity convergence term contribute to local circulation increases and the formation of coherent vortical structures. As shown by both our Eulerian and Lagrangian results, these maxima and minima in vorticity convergence occur at the convective line ends where the mesoscale vortices are observed.

\section{Summary and conclusions}

This study has examined the mechanisms responsible for the production, organization, and maintenance of vertical vorticity in the early stages of a simulation of an idealized, finite-length convective line in a westerly shear environment. Since previous simulations of similar midlatitude convective lines (DW94; SWK; Weisman and Davis 1998) were all performed using the Klemp-Wilhemson (1978) model, it is first of interest 


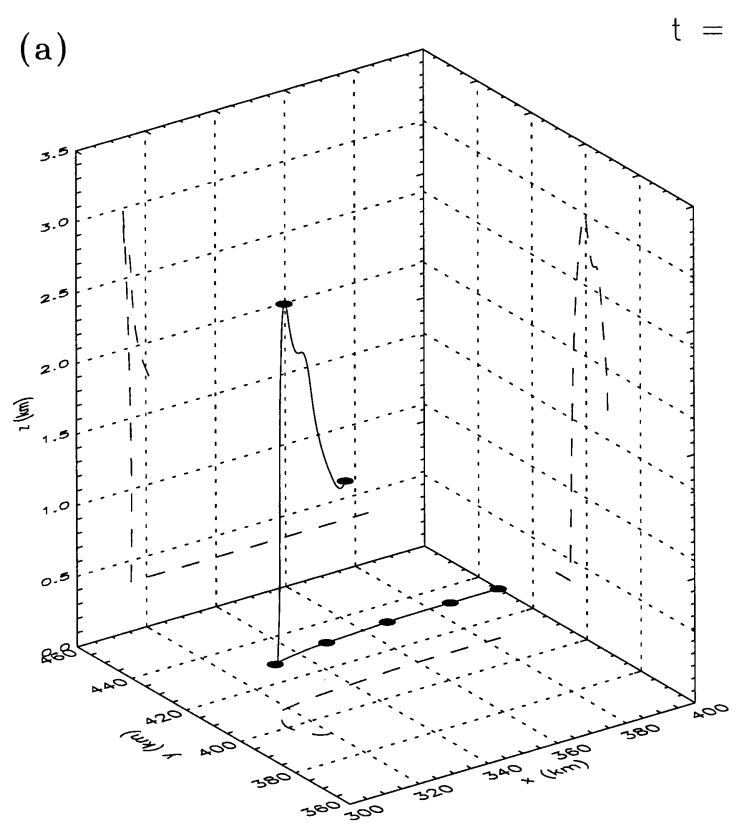

(c)

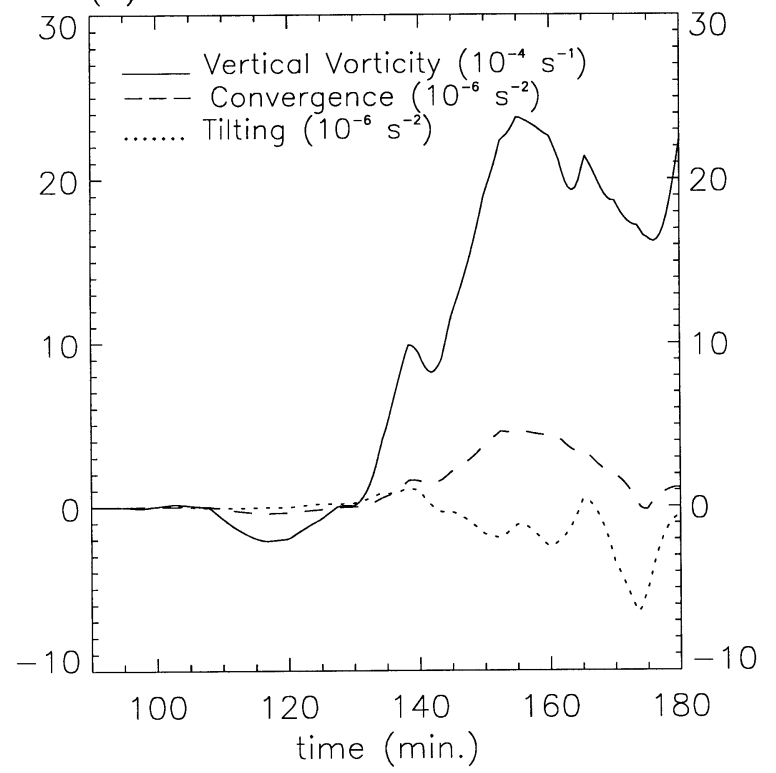

(b)

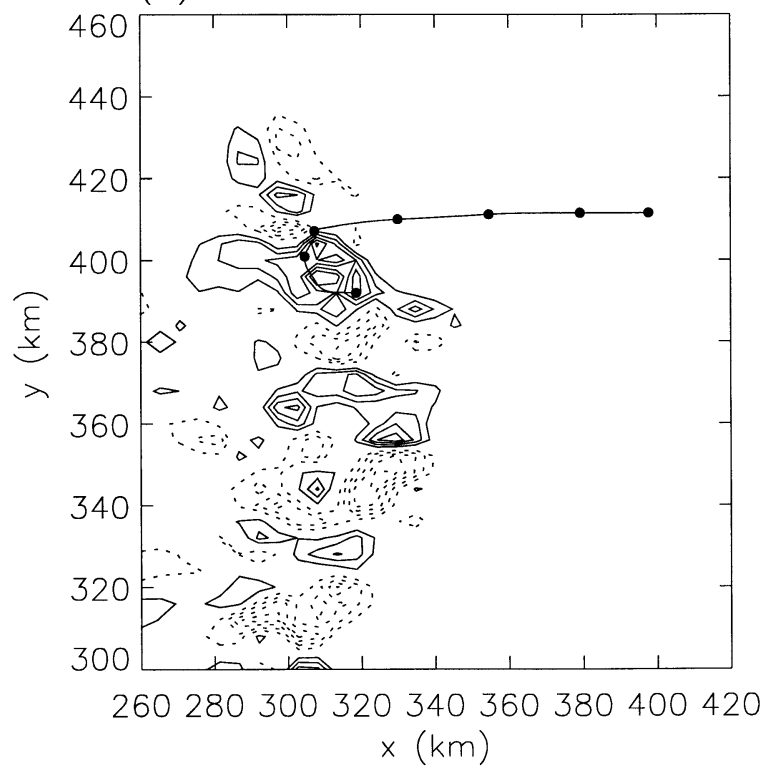

(d)

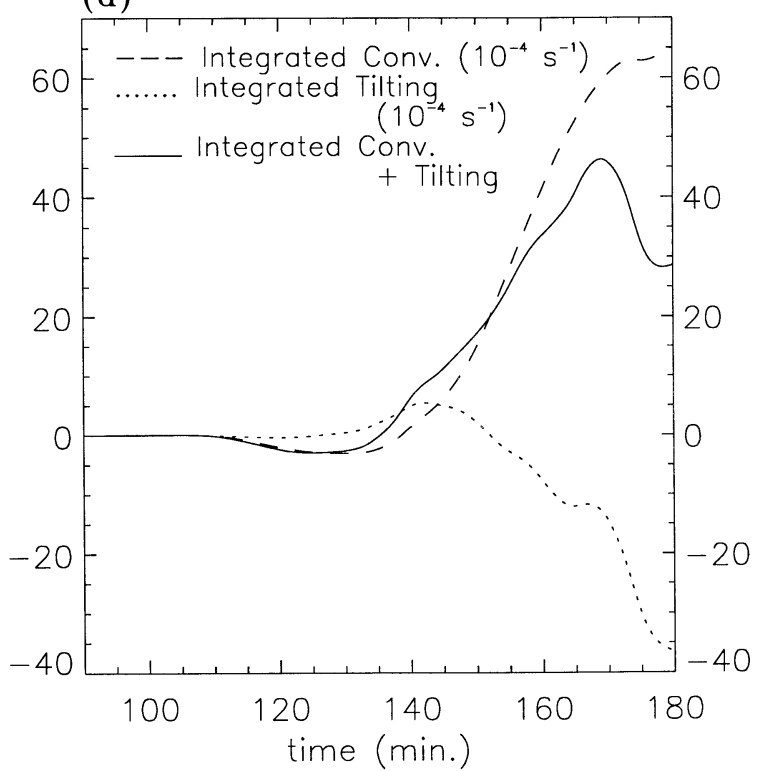

FIG. 8. Time-dependent back trajectory from $t=3 \mathrm{~h}$ to $t=0 \mathrm{~h}$ originating from the center of the northern vortex at $z=1.75 \mathrm{~km}$ : (a) three-dimensional trajectory with projections onto the two-dimensional planes (dashed lines); (b) horizontal trajectory superimposed on vertical vorticity contours at $z=1.75 \mathrm{~km}$ at $3 \mathrm{~h}$; (c) instantaneous values of vertical vorticity $\left(10^{-4} \mathrm{~s}^{-1}\right.$, solid line $)$, convergence tendency $\left(10^{-6} \mathrm{~s}^{-2}\right.$, dashed line), and tilting tendency $\left(10^{-6} \mathrm{~s}^{-2}\right.$, dotted line) calculated along the trajectory; (d) integrated convergence tendency $\left(10^{-4}\right.$ $\mathrm{s}^{-1}$, dashed line), integrated tilting tendency $\left(10^{-4} \mathrm{~s}^{-1}\right.$, dotted line), and the sum of integrated convergence and tilting tendencies (solid line) calculated along the trajectory. Time increments of $0.5 \mathrm{~h}$ are marked by solid dots on the trajectory. Contour increment of vertical vorticity is $5 \times 10^{-4} \mathrm{~s}^{-1}$, and the zero contour has been omitted.

to investigate the robustness of these results with a different cloud-resolving model. Using RAMS, the main features of the convective line (e.g., PV and vertical vorticity fields) shown in DW94 were reproduced. This is encouraging, and we can thus regard the principal flow features in all of these simulations as meaningful and model-independent.
An asymmetry in vertical vorticity occurs early and rapidly in the MCS lifecycle; it is for this reason we focus on the early stages of the formation and organization of MCVs. With no ambient vertical vorticity initially present, it is clear that vertical vorticity must come from tilting of positive ambient horizontal vorticity by the initial convective updrafts. Throughout the entire 
simulation, however, no single process appears to be dominant in organizing vertical vorticity. At early times in the simulation, tilting of ambient shear by convective updrafts and downdrafts associated with precipitation and the cold outflow provides the seed of vertical vorticity necessary for further development of the flow into MCVs. Although tilting of negative horizontal vorticity associated with the advancing cold pool observed in this simulation is consistent with Weisman and Davis (1998), they invoke this as the primary mechanism for generating organized mesoscale vortices after $3 \mathrm{~h}$. By $2 \mathrm{~h}$ into our simulation, tilting of negative storm-generated horizontal vorticity contributes to vertical vorticity production, but the systemwide tilting field is disorganized and no clear asymmetry is evident. Vorticity convergence, meanwhile, is weaker in magnitude than tilting but its sign and spatial extent indicate that it is contributing to the amplification of the asymmetry present in vertical vorticity. Similar structures in tilting and convergence were seen in SWK at $t=6 \mathrm{~h}$. A Lagrangian trajectory analysis of the tendency terms in the vertical vorticity equation, however, confirms that the vertical vorticity of air parcels reaching the Eulerian display level examined $(z=1.05 \mathrm{~km})$ have the consistent sign of tilting en route to the display level. The apparent inconsistency between tilting and vertical vorticity in the Eulerian plan views arises because the tilting of air parcels changes sign before reaching the display level. The vertical vorticity that is generated by tilting is not quickly diffused, thus vorticity convergence is able to enhance and organize the vorticity on the system scale.

The Lagrangian trajectory analysis confirms how vorticity tilting and convergence tendencies affect the rate of change of vertical vorticity following an air parcel at different times in the simulation. A term-by-term budget analysis of the vertical vorticity equation along parcel trajectories shows that vorticity tilting is dominant at early times, while vorticity convergence becomes a comparable contributor to vertical vorticity intensification and organization at $2 \mathrm{~h}$ and later. Tilting and convergence, therefore, assume a cooperative role in the generation and organization of MCVs. Vertical vorticity is produced by the tilting of both ambient and stormgenerated horizontal vorticity, while convergence then enhances the vorticity that is produced. These results are consistent with the tropical Lagrangian analysis of Trier et al. (1997) despite our midlatitude case having substantially different environmental conditions. Although the similarities in vorticity production between midlatitude and tropical squall lines has been noted previously (Weisman et al. 1993; Trier et al. 1997), they have not yet to our knowledge been confirmed by a comparative midlatitude squall line Lagrangian budget analysis as provided in the present study.

We are in full agreement with DW94 and Weisman and Davis (1998) that in the absence of ambient vertical vorticity the source of vertical vorticity in convective lines is due to tilting of either ambient or storm-gen- erated horizontal vorticity, or both. The phenomenological interpretation provided in their papers, however, bases not only the source, but also the organization and maintenance of the line-end mesoscale vortices primarily on tilting. As we show in our paper, one cannot invoke tilting alone to explain the organization of mesoscale vortices in a finite-extent squall line; one must also examine how vorticity convergence enhances and organizes the vertical vorticity that is produced by tilting.

The similarities in structure and lifetime between midlatitude and tropical MCVs enable us to apply what we have learned in the midlatitude case to further our understanding of the precursors supporting tropical cyclone formation (Zehr 1992). As discussed by Weisman et al. (1993) and Trier et al. (1997), the mechanisms that govern vertical vorticity generation and organization in tropical squall lines are the same that lead to MCVs in their midlatitude counterparts. Following Rotunno et al. (1988), Weisman et al. (1993) also note the importance of an upshear-tilted circulation in convective systems, which leads to the development of a midlevel MCV. The cold pool generated by such systems must be stronger than the ambient shear to produce the upshear-tilted structure. For tropical systems, this ultimately means that the ambient low-level shear must be weak, since tropical cold pools are weak in strength as well. The resultant MCV, given its long life and ability to trigger new convection, then creates an environment that is more conducive to tropical cyclogenesis (Montgomery and Enagonio 1998; Trier et al. 2000a,b; Enagonio and Montgomery 2001; Möller and Montgomery 2000; Davis and Bosart 2001). This topic is a subject of an ongoing investigation, which will be reported in due course.

Acknowledgments. The authors wish to thank Mr. John Persing for lending us his Lagrangian trajectory programming routines. The authors also greatly appreciate the helpful comments and improvements offered by the three anonymous reviewers. Discussions with Dr. David Nolan and Dr. Chris Davis are appreciated as well. This research was funded in part by the National Science Foundation under Grant ATM-9732678 and Colorado State University.

\section{REFERENCES}

Bosart, L. F., and F. Sanders, 1981: The Johnstown flood of July 1977 A long-lived convective storm. J. Atmos. Sci., 38, 1616-1642.

Davis, C. A., and W. L. Weisman, 1994: Balanced dynamics of mesoscale vortices produced in simulated convective systems. $J$. Atmos. Sci., 51, 2005-2030.

, and L. F. Bosart, 2001: Numerical simulations of the genesis of Hurricane Diana (1984). Part I: Control simulation. Mon. Wea. Rev., 129, 1859-1881.

Enagonio, J., and M. T. Montgomery, 2001: Tropical cyclogenesis via convectively forced vortex Rossby waves in a shallow water primitive equation model. J. Atmos. Sci., 58, 685-705.

Fujita, T. T., 1978: Manual of downburst identification for Project 
NIMROD. Satellite and Mesometeorology Research Paper 156, Dept. of Geophysical Sciences, University of Chicago, 104 pp.

Klemp, J. B., and R. B. Wilhelmson, 1978: The simulation of threedimensional convective storm dynamics. J. Atmos. Sci., 35, 1070-1096.

Mellor, G. L., and T. Yamada, 1974: Hierarchy of turbulence closure models for planetary boundary layers. J. Atmos. Sci., 31, 17911806.

Möller, J. D., and M. T. Montgomery, 2000: Tropical cyclone evolution via potential vorticity anomalies in a three-dimensional balance model. J. Atmos. Sci., 57, 3366-3387.

Mesinger, F., and A. Arakawa, 1976: Numerical Methods Used in Atmospheric Models. GARP Publication Series, Vol. 14, WMO/ ICSU Joint Organizing Committee, 64 pp.

Montgomery, M. T., and J. Enagonio, 1998: Tropical cyclogenesis via convectively forced Rossby waves in a three-dimensional quasi-geostrophic model. J. Atmos. Sci., 55, 3176-3207.

Pielke, R. A., and Coauthors, 1992: A comprehensive meteorological modeling system-RAMS. Meteor. Atmos. Phys., 49, 69-91.

Rotunno, R., J. B. Klemp, and M. L. Weisman, 1988: A theory for strong, long-lived squall lines. J. Atmos. Sci., 45, 463-485.

Skamarock, W. C., M. L. Weisman, and J. B. Klemp, 1994: Threedimensional evolution of simulated long-lived squall lines. $J$. Atmos. Sci., 51, 2563-2584.

Smagorinsky, J., 1963: General circulation experiments with the primitive equations. Part I: The basic experiment. Mon. Wea. Rev., 91, 91-164.

Trier, S. B., W. C. Skamarock, and M. A. LeMone, 1997: Structure and evolution of the 22 February 1993 TOGA COARE squall line: Organization mechanisms inferred from numerical simulation. J. Atmos. Sci., 54, 386-407.

- C. A. Davis, and J. D. Tuttle, 2000a: Long-lived mesoconvective vortices and their environment. Part I: Observations from the central United States during the 1998 warm season. Mon. Wea. Rev., 128, 3376-3395

- — - and W. C. Skamarock, 2000b: Long-lived mesoconvective vortices and their environment. Part II: Induced thermodynamic destabilization in idealized simulations. Mon. Wea. Rev., 128, 3396-3412.

Walko, R. L., W. R. Cotton, M. P. Meyers, and J. Y. Harrington, 1995 : New RAMS cloud microphysics parameterization. Part I: The single moment scheme. Atmos. Res., 38, 29-62.

Weisman, M. L., 1993: The genesis of severe, long-lived bow echoes. J. Atmos. Sci., 50, 645-670.

_- and C. A. Davis, 1998: Mechanisms for the generation of mesoscale vortices within quasi-linear convective systems. $J$. Atmos. Sci., 55, 2603-2622.

— W. W. Skamarock, and J. B. Klemp, 1993: The generation of mesoscale convective vortices within long-lived convective systems. Preprints, 20th Conf. on Hurricanes and Tropical Meteorology, San Antonio, TX, Amer. Meteor. Soc., 120-123.

Zehr, R., 1992: Tropical cyclogenesis in the western North Pacific. NOAA Tech. Rep. NESDIS 61, $181 \mathrm{pp}$.

Zhang, D. L., and J. M. Fritsch, 1987: Numerical simulation of the Meso- $\beta$ scale structure and evolution of the 1977 Johnstown flood. Part II: Inertially stable warm-core vortex and the mesoscale convective complex. J. Atmos. Sci., 44, 2593-2612. 